\title{
Síndrome de Guillain Barré e infección por SARS-CoV-2: reporte de dos casos en Perú.
}

\author{
Guillain Barré syndrome and SARS-CoV-2 infection: Report of two cases in Peru. \\ Darwin Segura-Chávez 1,a, Deysi Ordinola-Calle 2,b, Isabel Tagle-Lostaunau ${ }^{1, a}$, Francisco Aquino-Peña 1,a, \\ Juan Sifuentes-Monge ${ }^{1, a}$.
}

\section{RESUMEN}

La enfermedad por coronavirus 2019 (COVID-19), provocada por el virus SARS-CoV-2, se manifiesta principalmente a nivel respiratorio. Sin embargo, puede afectar otros órganos y sistemas, entre ellos el sistema nervioso, generando diversas manifestaciones neurológicas en las que se incluye el síndrome de Guillain Barré (SGB). Presentamos dos casos de pacientes varones de 35 y 46 años con SGB, uno clasificado como neuropatía axonal motora aguda (AMAN) y el otro como polineuropatia inflamatoria desmielinizante aguda (AIDP), quienes mostraron serología positiva para SARS-CoV-2 y sintomatología respiratoria previa al cuadro de debilidad. En ambos casos, la reacción en cadena de polimerasa en tiempo real (RT-PCR) en secreción faríngea y liquido cefalorraquideo fue negativa. El diagnóstico de infección por SARS-CoV-2 fue confirmado por prueba de anticuerpos totales en suero, mediante inmunoensayo CLIA (Chemiluminescent Immunoassay), positiva para IgG en ambos casos. Las características de los casos presentados sustentan una posible relación posinfecciosa entre el SARS-CoV-2 y el SGB y abren, además, la posibilidad de que, a pesar de que los pacientes sean asintomáticos o presenten síntomas respiratorios leves, podrían desarrollar complicaciones posinfecciosas tales como SGB.

PALABRAS CLAVE: COVID-19, Polirradiculoneuropatía Aguda Inflamatoria, Síndrome de Guillain Barré.

\section{SUMMARY}

Coronavirus disease 2019 (COVID-19), caused by the SARS-CoV-2 virus, shows mainly clinical manifestations at the respiratory level. It can affect, however, other organs and systems, including the central nervous system, generating neurological manifestations that include the Guillain Barré Syndrome (GBS). We present the cases of two male patients, aged 35 and 46, with GBS, one classified as AMAN and the other as AIDP. Both patients showed positive serology for SARS-CoV-2 and reported respiratory symptoms prior to the weakness condition. RT-PCR in pharyngeal secretion and CSF was negative in both cases. The infection by SARS-CoV- 2 was confirmed by the total antibody test in serum through a positive CLIA immunoassay, positive for IgG in the two cases. The characteristics of the cases support a possible postinfectious relationship between SARS-CoV-2 and GBS, and open as well the possibility that despite being asymptomatic or having mild respiratory symptoms, some patients may develop postinfectious complications such as GBS.

KEYWORDS: COVID-19, Acute Inflammatory Polyneuropathy, Guillain Barré syndrome.

1 Departamento de Neurofisiología Clínica, Unidad de Investigación en Enfermedades Neuromusculares, Instituto Nacional de Ciencias Neurológicas. Lima, Perú.

2 Instituto Nacional de Ciencias Neurológicas. Lima, Perú.

a Médico Neurólogo; ${ }^{\mathrm{b}}$ Médico residente de Neurología. 


\section{INTRODUCCIÓN}

La enfermedad por coronavirus 2019 (COVID-19), provocada por la infección del coronavirus tipo 2 causante del síndrome respiratorio agudo grave (SARS-CoV-2), originada en la ciudad de Wuhan, China, se ha constituido en el peor desastre sanitario del último siglo, sólo comparable a la pandemia de gripe española de $1918(1,2)$.

El SARS-CoV-2 genera un trastorno multisistémico que se manifiesta principalmente a nivel respiratorio generando síntomas como tos seca, disnea, dolor torácico y en casos severos insuficiencia respiratoria que puede requerir ventilación mecánica (1); su diagnóstico se realiza a través de pruebas moleculares de detección de ARN viral mediante técnicas de reacción en cadena de la polimerasa en tiempo real (RT-PCR); así como, con pruebas serológicas de detección de anticuerpos contra antígenos virales, las cuales detectan dos tipos de inmunoglobulinas (Ig), $\operatorname{IgM}$ e IgG que se producen en promedio 5 y 14 días después de la infección respectivamente (2).

Cada vez existen más reportes de manifestaciones neurológicas asociadas a la infección del SARS-CoV-2, clasificadas como manifestaciones relacionadas con la neuroinvasión e infección directa del virus, manifestaciones neurológicas inespecíficas de carácter sistémico, complicaciones cerebrovasculares y complicaciones inmunomediadas que afectan el sistema nervioso central y periférico en la que se incluye el síndrome de Guillain Barré (SGB) (3).

El SGB es una polirradiculoneuropatia aguda de causa autoinmune, que en un $70 \%$ de casos está precedido en 1 a 2 semanas de una infección respiratoria o gastrointestinal (4). Clásicamente el SGB se ha asociado a múltiples disparadores infecciosos, recientemente el virus SARS-CoV-2, se ha relacionado con SGB y los reportes son cada vez más abundantes al respecto $(5,6)$.

Presentamos el caso de dos pacientes con SGB, y serología positiva para SARS CoV-2 quienes mostraron un perfil posinfeccioso, típicamente relacionado con SGB.

\section{Caso 1}

Varón de 35 años que inició enfermedad con debilidad en miembro superior izquierdo, posteriormente (un día después) comprometió miembro superior derecho y miembros inferiores. La debilidad se intensificó progresivamente hasta impedirle la bipedestación y la marcha, acudiendo por emergencia 9 días después del inicio de los síntomas. Dentro de los antecedentes refirió que dos semanas previas al inicio de la debilidad presentó tos seca, dolor de garganta, congestión nasal y alza térmica durante 5 días.

El examen físico reveló un paciente afebril, lúcido, con facies simétrica, sin dificultad respiratoria, la evaluación de fuerza muscular mostró cuadriparesia con una escala de Medical Research Council (MRC) de $2 / 5$ a nivel proximal y $1 / 5$ a nivel distal en las extremidades superiores e inferiores, con sensibilidad táctil y vibratoria conservada y arreflexia. Escala de discapacidad de Hughes: 4.

Los exámenes de laboratorio al ingreso mostraron: Leucocitos: 4,42 x 10 13/ul; Hemoglobina 14,4 mg/dl; Plaquetas $282 \times 10^{\wedge} / \mathrm{ul}$; Glucosa: $110 \mathrm{mg} / \mathrm{dl}$ Urea: $32 \mathrm{mg} /$ $\mathrm{dl}$; creatinina: $0,82 \mathrm{mg} / \mathrm{d}$; Prueba serológica rápida para SARS-CoV-2: Ig M: reactivo/ Ig G: reactivo; ELISA VIH I/II: No reactivo; LCR: proteínas:141 $\mathrm{mg} \%$, células: $03 \times$ campo, la tomografía de tórax no mostró anormalidades (tabla 1).

Durante su hospitalización se le solicitó anticuerpo antigangliósido GM1 el cual resultó negativo, la electromiografía fue compatible con neuropatía axonal motora aguda (AMAN) (tabla 2). El RT-PCR-SARS$\mathrm{CoV}-2$ en secreción faríngea y LCR resultó negativo, pero la prueba de anticuerpos totales para SARS-CoV-2 mediante inmunoensayo CLIA (ChemiLuminescent Immuno Assay), fue positiva para $\mathrm{IgG}$ con un título de 19,14 .96 AU/ml ( VN <10 AU/ml).

Recibió tratamiento con Inmunoglobulina intravenosa en dosis de $0,4 \mathrm{~g} / \mathrm{kg} /$ día durante 5 días, con lo cual el paciente evolucionó favorablemente. Al alta su escala de discapacidad de Hughes fue 3 puntos y su Erasmus GBS Outcome Score (EGOS) fue 4 puntos.

\section{Caso 2}

Varón de 46 años que inició enfermedad con debilidad en miembros inferiores que limitó la marcha y bipedestación en los primeros 4 días, luego comprometió también miembros superiores. Un día antes del ingreso, se agregó dificultad para deglutir y sensación de falta de aire, por lo cual acudió por emergencia a los 8 días de iniciado los síntomas. 
Segura-Chávez D, et al.

Tabla 1. Características clínicas de dos pacientes con Síndrome de Guillain Barré e Infección por SARS-CoV-2.

\begin{tabular}{|c|c|c|c|c|c|c|c|c|}
\hline $\begin{array}{l}\text { Nro. } \\
\text { Paciente }\end{array}$ & $\begin{array}{l}\text { Síndrome } \\
\text { respiratorio } \\
\text { previo }\end{array}$ & $\begin{array}{l}\text { Periodo } \\
\text { asintomático }\end{array}$ & $\begin{array}{l}\text { Signos y } \\
\text { síntomas } \\
\text { neurológicos }\end{array}$ & $\begin{array}{l}\text { Hallazgos en } \\
\text { LCR }\end{array}$ & $\begin{array}{l}\text { Anticuerpos } \\
\text { anti- } \\
\text { gangliósidos }\end{array}$ & $\begin{array}{l}\text { TAC } \\
\text { Tórax }\end{array}$ & $\begin{array}{l}\text { Clasificación } \\
\text { neurofisiológica }\end{array}$ & $\begin{array}{l}\text { Tratamiento y } \\
\text { estado a las } 4 \\
\text { semanas }\end{array}$ \\
\hline 1 & $\begin{array}{l}\text { Si, Fiebre, Dolor } \\
\text { faríngeo, } \\
\text { congestión } \\
\text { nasal, } 14 \\
\text { días previos } \\
\text { (duración } 5 \text { d) }\end{array}$ & 9 días & $\begin{array}{l}\text { Cuadriparesia } \\
\text { flácida } \\
\text { arreflexica. } \\
8 \text { días de } \\
\text { evolución }\end{array}$ & $\begin{array}{l}\text { Proteínas: } 141 \\
\text { mg\%, células: } \\
\text { 03 x campo } \\
\text { RT-PCR } \\
\text { en LCR: } \\
\text { negativo }\end{array}$ & $\begin{array}{l}\text { GM1: } \\
\text { Negativo }\end{array}$ & Normal & AMAN & $\begin{array}{l}\text { IGIV } 0.4 \mathrm{~g} / \mathrm{kg} \mathrm{x} \\
5 \text { días } \\
\text { Evolución } \\
\text { Favorable } \\
\text { EGOS al alta :4 }\end{array}$ \\
\hline 2 & $\begin{array}{l}\mathrm{Si} \text {, Cefalea, } \\
\text { Tos seca y alza } \\
\text { térmica, } 10 \\
\text { días previos } \\
\text { (duración } 7 \text { días) }\end{array}$ & 3 días & $\begin{array}{l}\text { Cuadriparesia } \\
\text { flácida } \\
\text { arreflexica }+ \\
\text { diparesia facial } \\
8 \text { días de } \\
\text { evolución }\end{array}$ & $\begin{array}{l}\text { Proteínas: } 228 \\
\text { mg\%, células: } \\
03 \text { x campo } \\
\text { RT-PCR: } \\
\text { negativo }\end{array}$ & No realizado & Normal & AIDP & $\begin{array}{l}\text { IGIV } 0.4 \mathrm{~g} / \mathrm{kg} \text { x } \\
5 \text { días } \\
\text { Evolución } \\
\text { favorable. } \\
\text { EGOS al alta:3.5 }\end{array}$ \\
\hline
\end{tabular}

LCR: liquidocefalorraquideo, RT-PCR: reacción en cadena de polimerasa en tiempo real; AMAN: neuropatia axonal motora aguda; AIDP: polirradiculopatia inflamatoria desmielinizante aguda; IGIV: inmunoglobulina intravenosa; GM1: gangliósido monosiálico 1; EGOS: Erasmus GBS Outcome Score

Tabla 2. Potencial de acción motor y sensitivo de cuatro extremidades del caso 1 .

\begin{tabular}{|c|c|c|c|c|c|c|}
\hline \multicolumn{7}{|c|}{ ESTUDIO DE CONDUCCION MOTORA } \\
\hline \multirow[t]{2}{*}{ NERVIO } & \multicolumn{2}{|c|}{$\begin{array}{l}\text { LATENCIA MOTORA } \\
\text { DISTAL, ms }\end{array}$} & \multicolumn{2}{|c|}{ AMPLITUD, mV } & \multicolumn{2}{|c|}{$\begin{array}{l}\text { VELOCIDAD DE } \\
\text { CONDUCCION, m/sec }\end{array}$} \\
\hline & $\begin{array}{l}\text { Valores } \\
\text { Normales }\end{array}$ & Valores & $\begin{array}{l}\text { Valores } \\
\text { Normales }\end{array}$ & Valores & $\begin{array}{l}\text { Valores } \\
\text { Normales }\end{array}$ & Valores \\
\hline Facial, Der/Izq. & $<4,0 \mathrm{~ms}$ & $\mathrm{NR} / \mathrm{NR}$ & $>1,0 \mathrm{mV}$ & NR/NR & & \\
\hline Mediano, Der/Izq. & $<3,8 \mathrm{~ms}$ & NR/NR & $>3,5 \mathrm{mV}$ & NR/NR & $>50 \mathrm{~m} / \mathrm{seg}$ & $\mathrm{NR} / \mathrm{NR}$ \\
\hline Cubital, Der/Izq. & $<3,8 \mathrm{~ms}$ & NR/NR & $>3,5 \mathrm{mV}$ & NR/NR & $>50 \mathrm{~m} / \mathrm{seg}$ & $\mathrm{NR} / \mathrm{NR}$ \\
\hline Peroneal, Der/Izq. & $<5,5 \mathrm{~ms}$ & NR/NR & $>2,5 \mathrm{mV}$ & NR/NR & $>40 \mathrm{~m} / \mathrm{seg}$ & $\mathrm{NR} / \mathrm{NR}$ \\
\hline Tibial, Der/izq. & $<5,5 \mathrm{~ms}$ & $\mathrm{NR} / \mathrm{NR}$ & $>2,5 \mathrm{mV}$ & $\mathrm{NR} / \mathrm{NR}$ & $>40 \mathrm{~m} / \mathrm{seg}$ & $\mathrm{NR} / \mathrm{NR}$ \\
\hline \multicolumn{7}{|c|}{ ESTUDIO DE CONDUCCION SENSITIVA } \\
\hline \multirow[t]{2}{*}{ NERVIO } & \multicolumn{2}{|c|}{ LATENCIA } & \multicolumn{2}{|l|}{ AMPLITUD } & \multicolumn{2}{|c|}{ VELOCIDAD } \\
\hline & $\begin{array}{l}\text { Valores } \\
\text { normales } \\
\end{array}$ & Valores & $\begin{array}{l}\text { Valores } \\
\text { normales } \\
\end{array}$ & Valores & $\begin{array}{l}\text { Valores } \\
\text { normales } \\
\end{array}$ & Valores \\
\hline Mediano Der/Izq. & $<3,2 \mathrm{~ms}$ & $3,0 / 2,8$ & $>20 \mathrm{mV}$ & $22 / 26$ & $>50 \mathrm{~m} / \mathrm{seg}$ & - \\
\hline $\begin{array}{l}\text { Sural } \\
\text { Der/Izq. }\end{array}$ & $<4,0 \mathrm{~ms}$ & $2,7 / 2,5$ & $>20 \mathrm{mV}$ & $25 / 23$ & $>50 \mathrm{~m} / \mathrm{seg}$ & - \\
\hline
\end{tabular}

NR: No Registrado o Ausente.

ms: milisegundos

$\mathrm{mV}$ : milivoltios

Refirió que 10 días previos al inicio de la debilidad cursó con cefalea, tos seca y alza térmica durante 7 días.

El examen físico reveló un paciente afebril, lúcido, con diparesia facial, sin dificultad respiratoria. La evaluación de fuerza muscular mostró cuadriparesia (MRC de 4/5 en los segmentos proximales de extremidades superiores y $3 / 5$ en porciones distales de las extremidades superiores y en las extremidades inferiores), sensibilidad táctil y vibratoria disminuida y arreflexia. Escala de discapacidad de Hughes: 4 y Erasmus GBS Respiratory Insufficiency Score (EGRIS): 3 puntos.

Los exámenes de laboratorio al ingreso mostraron:

Leucocitos: 11,37 x 10^3/ul; Hemoglobina 15,7 $\mathrm{mg} / \mathrm{dl}$; Plaquetas $298 \times 10^{\wedge 3} / \mathrm{ul}$; Glucosa: $120 \mathrm{mg} /$ dl; Urea:21 mg/dl; creatinina: 0,64 mg/dl; Prueba 
Síndrome de Guillain Barré e infección por SARS-COV-2: reporte de dos casos en Perú.

Tabla 3. Potencial de acción motor y sensitivo de cuatro extremidades del caso 2.

\begin{tabular}{|c|c|c|c|c|c|c|}
\hline \multicolumn{7}{|c|}{ ESTUDIO DE CONDUCCION MOTORA } \\
\hline \multirow[t]{2}{*}{ NERVIO } & \multicolumn{2}{|c|}{$\begin{array}{l}\text { LATENCIA MOTORA } \\
\text { DISTAL, ms }\end{array}$} & \multicolumn{2}{|c|}{ AMPLITUD, mV } & \multicolumn{2}{|c|}{$\begin{array}{l}\text { VELOCIDAD DE } \\
\text { CONDUCCION, m/sec }\end{array}$} \\
\hline & $\begin{array}{l}\text { Valores } \\
\text { Normales }\end{array}$ & Valores & $\begin{array}{l}\text { Valores } \\
\text { Normales }\end{array}$ & Valores & $\begin{array}{l}\text { Valores } \\
\text { Normales } \\
\end{array}$ & Valores \\
\hline Mediano, Der/Izq. & $<3,8 \mathrm{~ms}$ & $14,7 / 13,4$ & $>3,5 \mathrm{mV}$ & $2,9 / 1,1$ & $>50 \mathrm{~m} / \mathrm{seg}$ & $31,4 / 38,8$ \\
\hline Cubital, Der/Izq. & $<3,8 \mathrm{~ms}$ & $12,8 / 12,2$ & $>3,5 \mathrm{mV}$ & $3,1 / 2,8$ & $>50 \mathrm{~m} / \mathrm{seg}$ & $35,1 / 34,2$ \\
\hline Peroneal, Der/Izq. & $<5,5 \mathrm{~ms}$ & $20,5 / 16,0$ & $>2,5 \mathrm{mV}$ & $1,4 / 1,3$ & $>40 \mathrm{~m} / \mathrm{seg}$ & $27,8 / 28,2$ \\
\hline Tibial, Der/izq. & $<5,5 \mathrm{~ms}$ & $24,5 / 20,9$ & $>2,5 \mathrm{mV}$ & $1,2 / 1,0$ & $>40 \mathrm{~m} / \mathrm{seg}$ & $26,0 / 26,3$ \\
\hline \multicolumn{7}{|c|}{ ESTUDIO DE CONDUCCION SENSITIVA } \\
\hline \multirow[t]{2}{*}{ NERVIO } & \multicolumn{2}{|l|}{ LATENCIA } & \multicolumn{2}{|l|}{ AMPLITUD } & \multicolumn{2}{|c|}{ VELOCIDAD } \\
\hline & $\begin{array}{l}\text { Valores } \\
\text { normales }\end{array}$ & Valores & $\begin{array}{l}\text { Valores } \\
\text { normales }\end{array}$ & Valores & $\begin{array}{l}\text { Valores } \\
\text { normales }\end{array}$ & Valores \\
\hline Mediano Der/Izq. & $<3,2 \mathrm{~ms}$ & $\mathrm{NR} / \mathrm{NR}$ & $>20 \mathrm{mV}$ & $\mathrm{NR} / \mathrm{NR}$ & $>50 \mathrm{~m} / \mathrm{seg}$ & - \\
\hline $\begin{array}{l}\text { Sural } \\
\text { Der/Izq. }\end{array}$ & $<4,0 \mathrm{~ms}$ & $4,2 / 3,0$ & $>20 \mathrm{mV}$ & $7,3 / 6,9$ & $>50 \mathrm{~m} / \mathrm{seg}$ & - \\
\hline
\end{tabular}

NR: No Registrado o Ausente.

ms: milisegundos

$\mathrm{mV}$ : milivoltios

serológica rápida para SARS-CoV-2: Ig M: reactivo/ Ig G: reactivo; ELISA VIH I/II: No reactivo; LCR: proteínas: $228 \mathrm{mg} \%$, células: $03 \times$ campo, la tomografía de tórax no mostró anormalidades (tabla 1).

La electromiografía fue compatible con polineuropatia inflamatoria desmielinizante aguda (AIDP) (tabla 3), el RT-PCR para SARS-CoV-2 en secreción faríngea y LCR fue negativo, pero la prueba de anticuerpos totales para SARS-CoV-2 mediante inmunoensayo CLIA, fue positiva para $\operatorname{IgG}$ con un título de 86,96 AU/ml (VN $<10 \mathrm{AU} / \mathrm{ml})$

Recibió tratamiento con Inmunoglobulina intravenosa en dosis de $0,4 \mathrm{~g} / \mathrm{kg} /$ día durante 5 días, con lo cual el paciente evolucionó favorablemente. La escala de discapacidad de Hughes al alta fue 3 puntos y el EGOS fue 3,5 puntos.

\section{DISCUSIÓN}

Los casos descritos corresponden a dos pacientes varones de 35 y 46 años que cumplieron con los criterios clínicos y neurofisiológicos para SGB, el primero se clasificó como AMAN y el segundo como AIDP (4), ambos pacientes manifestaron un cuadro clínico caracterizado por cuadriparesia flácida arrefléxica, el segundo caso además presentó diparesia facial, ninguno evolucionó a falla respiratoria, ni enfermedad pulmonar grave.

Los pacientes refirieron síntomas respiratorios leves autolimitados que precedieron en 14 y 10 días respectivamente al inicio de la debilidad, mostrando el patrón típico posinfeccioso visto en SGB (7).

Diferentes agentes infecciosos en donde se incluye el citomegalovirus, virus de Epstein-Barr, virus de la inmunodeficiencia humana, virus del Zika, Haemophilus influenzae, campylobacter jejuni y otros, como los coronavirus OC43 y MERS-CoV, se han relacionado como disparadores de este síndrome (8), aunque todos estos no fueron descartados en los pacientes, hubo evidencia serológica de infección por SARS-CoV-2, con el cual diversos reportes recientes ya han postulado una relación causal posinfecciosa $(5,6)$.

En los pacientes el diagnóstico de infección por SARS-CoV-2 se sustentó en la presencia de anticuerpos $\operatorname{IgM}$ e $\operatorname{IgG}$ contra este virus en suero; aunque no existió un estudio para SARS-CoV-2 
previo que sustente la temporalidad entre la infección por este virus y el SGB, la presencia de IgG al ingreso, momento en el cual los pacientes sólo tenían 8 días de evolución del cuadro neurológico, podría indicar que los pacientes estuvieron infectados previo al inicio del SGB y que esta infección fue probablemente también la causa de los síntomas respiratorios en ambos pacientes, ya que para que la $\operatorname{IgG}$ esté presente debieron haber transcurrido aproximadamente 14 días luego de la infección (2); si bien es cierto el RT-PCR para SARS-CoV-2 en secreción faríngea, realizada días después del ingreso, resultó negativo en ambos casos, el diagnostico de infección por SARS-CoV-2 fue confirmado a través de la prueba de detección de anticuerpos totales mediante inmunoensayo CLIA las cuales mostraron títulos positivos para IgG. El intervalo de tiempo entre el inicio de los síntomas respiratorios y el inicio del SGB, en ambos pacientes coincide, con la mayoría de casos reportados hasta el momento que informan periodos entre 5 y 15 y en algunos casos de hasta 24 días $(7,9,10)$ dos revisiones sistemáticas consecutivas que evaluaron las características clínicas y paraclínicas de 24 y 30 pacientes con SGB e infección por SARS-CoV-2, han informado resultados similares. En ambas revisiones el AIDP fue el subtipo más frecuente, además se encontró que en la mayoría de los casos en que los anticuerpos antigangliosidos fueron estudiados, el resultado fue negativo, con excepción de uno que informó la presencia del anticuerpo anti-GD1b en un caso con síndrome de Miller Fisher e infección por SARS-CoV-2 (11-13). En nuestro reporte, sólo al primer caso se le realizó el dosaje del anticuerpo anti-GM1 el cual resulto negativo, cabe señalar que, a diferencia de la mayoría de estudios previos, no realizamos el estudio de otros anticuerpos antigangliosidos.

En concordancia con mayoría de casos reportados, nuestros pacientes no presentaron evidencia de infección por SARS-CoV-2 en las muestras de LCR, lo cual alejó la posibilidad de daño directo del virus como mecanismo patogénico y apoyó junto al perfil temporal de los síntomas un mecanismo posinfeccioso probablemente autoinmune (10-12).

Al igual que en otros reportes los pacientes mostraron buena respuesta clínica, luego del tratamiento con inmunoglobulina intravenosa en dosis de $0,4 \mathrm{~g} / \mathrm{Kg} /$ día por 5 dias, ambos al alta mejoraron su puntaje Hughes en un grado con respecto al ingreso, ninguno necesitó un curso adicional de inmunoglobulinas y/o plasmaféresis (10-12).

Nuestro reporte, muestra dos casos de SGB e infección por SARS-CoV-2, en los que sus características como la relación temporal entre los síntomas, la ausencia de evidencia de la presencia del virus en LCR y por tanto de daño directo sobre el sistema nervioso, y la buena respuesta al tratamiento con inunoglobulina, sustentan la hipótesis de una probable relación inmunológica posinfecciosa, además según lo observado en nuestros casos se expone la posibilidad, que a aún cuando los pacientes con infección por SARS-CoV-2, hayan sido asintomáticos o hayan presentado síntomas respiratorios leves, podrían desarrollar complicaciones posinfecciosas como el SGB, Sin embargo ambas observaciones aunque son cada vez más aceptadas, deben seguir siendo evaluadas en estudios posteriores que incluyan una mayor cantidad de pacientes, lo cual constituye una limitante del presente reporte.

\section{Correspondencia}

Darwin Alberto Segura Chávez.

Dirección: Jr. Ancash 1271 Barrios Altos, Lima.

Teléfono: 51981890390

Correo electrónico: darwin1854@hotmail.com

Declaración de financiamiento y de conflicto de intereses:

Financiamiento: La investigación fue autofinanciada por los autores.

Conflictos de interés: Todos los autores declaran no tener ningún conflicto de interés,

Recibió la aprobación del Comité de Ética del Instituto Nacional de Ciencias Neurológicas

\section{REFERENCIAS BIBLIOGRÁFICAS}

1. Deng S-Q, Peng H-J. Characteristics of and Public Health Responses to the Coronavirus Disease 2019 Outbreak in China. J Clin Med. 2020;9(2):575. doi: $10.3390 / \mathrm{jcm} 9020575$

2. Rashid ZZ, Othman SN, Samat MNA, Kalsom U, Ken WK. Diagnostic performance of COVID-19 serology assays. Malays J Pathol. 2020;42(1):13-21.

3. Carod-Artal FJ. Neurological complications of coronavirus and COVID-19. Rev Neurol. 2020;70(9):311-22. doi:10.33588/rn.7009.2020179

4. Donofrio PD. Guillain-Barré Syndrome. Contin Minneap Minn. 2017;23(5):1295-309. doi: 10.1212/ con.0000000000000513

5. Sedaghat Z, Karimi N. Guillain Barre syndrome associated with COVID-19 infection: A case report. 
J Clin Neurosci. 2020;76:233-235. doi: 10.1016/j. jocn.2020.04.062

6. El Otmani H, El Moutawakil B, Rafai M-A, El Benna N, El Kettani C, Soussi M, et al. Covid-19 and Guillain-Barré syndrome: More than a coincidence! Rev Neurol (Paris). 2020;176(6):518-519. doi: 10.1016/j.neurol.2020.04.007

7. Camdessanche J, Morel J, Pozzetto B, Paul S, Tholance Y, Botelho-Nevers E. COVID-19 may induce Guillain-Barré syndrome. Rev Neurol (Paris). 2020; 176(6): 516-518. doi: 10.1016/j. neurol.2020.04.003

8. Whittaker A, Anson M, Harky A. Neurological Manifestations of COVID-19: A systematic review and current update. Acta Neurol Scand. 2020;142(1):14-22. doi:10.1111/ane.13266

9. Coen M, Jeanson G, Culebras-Almeida LA, Hübers A, Stierlin F, Najjar I, et al. Guillain-Barré syndrome as a complication of SARS-CoV-2 infection. Brain Behav Immun. 2020;87:111-112. doi: 10.1016/j. bbi.2020.04.074

10. Toscano G, Palmerini F, Ravaglia S, Ruiz L,
Invernizzi $\mathrm{P}$, Cuzzoni MG, et al. Guillain-Barré Syndrome Associated with SARS-CoV-2. N Engl J Med. 2020; 382(26):2574-2576. doi: 10.1056/ NEJMc200919

11. Finsterer J, Scorza FA, Ghosh R. COVID-19 polyradiculitis in 24 patients without SARS-CoV-2 in the cerebro-spinal fluid. J Med Virol. 2020;10.1002/ jmv.26121. doi: 10.1002/jmv.2612

12. Trujillo LM, Valenzuela SN, von Oetinger A, Relación entre COVID-19 y síndrome de GuillainBarré en adultos. Revisión sistemática. Neurologia. 2020;35(9):646-654. doi: 10.1016/j.nrl.2020.07.004

13. Gutiérrez-Ortiz C, Méndez A, Rodrigo-Rey S, San Pedro-Murillo E, Bermejo-Guerrero L, Gordo-Mañas $\mathrm{R}$, et al. Miller Fisher Syndrome and polyneuritis cranialis in COVID-19. Neurology. 2020;95(5): e601-e605. doi: 10.1212/WNL.0000000000009619

Recibido: 26/10/2020 Aceptado: 03/03/2021 